\title{
Adaptive Display Power Management for OLED Displays
}

\author{
Tan Kiat Wee \\ School of Information Systems \\ Singapore Management University \\ 80 Stamford Road, Singapore \\ kwtan.2010@phdis.smu.edu.sg
}

\author{
Rajesh Krishna Balan \\ School of Information Systems \\ Singapore Management University \\ 80 Stamford Road, Singapore \\ rajesh@smu.edu.sg
}

\begin{abstract}
Mobile gaming has become increasingly popular in the past few years with the proliferation of smartphones that have the increased CPU, memory, and network (3.5G etc.) capabilities to support a vast range of interesting games. In addition, these phones also have high quality displays, such as Organic Light Emitting Diodes (OLED) displays, that allow the intricate details in games to be shown in vivid detail to end users. Unfortunately, these displays tend to consume a lot of energy - which in turn limits the amount of time that a user can spend actually playing games on these devices. In this paper, we describe a technique that makes use of saliency, with respect to the end user, to reduce the power consumption of OLED displays when they are used for games, by reducing the brightness of game areas that are not of interest currently to the game player. We evaluated our technique with a user study and showed that even for fast-paced shooting games, our solution is able to save a modest amount of power with no impact in the end user gaming experience.
\end{abstract}

\section{Categories and Subject Descriptors}

K.8.0 [General]: Games; I.4.3 [Image Processing \& Computer Vision]: Enhancement; I.3.3 [Computer Graphics]: Picture / Image Generation-Display Algorithms

\section{General Terms}

Algorithms, Design, Human Factors, Experimentation

\section{Keywords}

Power Management, Saliency, Mobile Games

This work is supported in part by the Singapore Ministry of Education Academic Research Fund Tier 2 under the research grant T208B2301. Any opinions, findings, conclusions or recommendations expressed in this material are those of the authors and do not necessarily reflect the views of the granting agency, or Singapore Management University.

Permission to make digital or hard copies of all or part of this work for personal or classroom use is granted without fee provided that copies are not made or distributed for profit or commercial advantage and that copies bear this notice and the full citation on the first page. To copy otherwise, or republish, to post on servers or to redistribute to lists, requires prior specific permission and/or a fee.

MobiGames'12, August 13, 2012, Helsinki, Finland.

Copyright 2012 ACM 978-1-4503-1487-9/12/08... \$15.00.

\section{INTRODUCTION}

The proliferation of smartphones [10] has created and in turn been driven by the abundance of applications [9] available for the various smartphone platforms such as Apple's iPhone, Google's Android, and Windows Phone 7. One application category that is usually the most downloaded is mobile games [9].

However, even though games tend to be the most downloaded applications, paradoxically, they are also the hardest to support on smartphones as they demand the most resources - making use of several resources, such as computation, display, network, audio and interaction, at the same time. From our previous work [1], we noted that the display is one of the main power consuming components on mobile devices. However, our prior work focused solely on reducing the power usage of Liquid Crystal Displays (LCD). In this work, we focus on the other common mobile display; namely Organic Light Emitting Diodes (OLED) displays.

OLEDs, unlike LCDs, do not used any form of active backlighting technology. Instead OLEDs use a much more power-efficient display method that turns on and off individual LED elements directly. However, even though OLEDs are more power efficient than LCDs, there is still room for improvement. For example, prior work [8, 3] has shown that manipulating what is being displayed can greatly improve the energy efficiency of OLED displays. In our work, we focused on an orthogonal approach by integrating the concept of saliency into the power management framework. Saliency refers to how much a particular object, environment, or scene stands out against the surrounding objects, environments, or scenes.

The main contribution of this paper is that we integrate this notion of saliency into a power management framework for OLED displays. In particular, we first identify the parts of the currently displayed image that are of highest interest to the user. We then save power by gradually dimming the non-interesting regions of the display.

We implemented and tested our solution on a Nexus One Android phone using a fast first-person shooting game called Kwaak 3 [11]. We then tested our solution via a user study involving 30 participants. Our results showed that our approach can save up to $11 \%$ of the display power (which translates to a $4 \%$ overall power savings) with minimal impact on end user perception.

Even though the overall power savings are low, we think that this is a promising approach that can complement the prior work that focuses on altering the content itself to match the properties of the OLED displays. 


\section{RELATED WORK}

OLED and Active Matrix OLED (AMOLED) displays in smartphones have the characteristic where each pixel is emissive (i.e., each pixel can display one of three colors red, green, or blue). This emissive nature eliminates the need for the energy-consuming backlight component found in LCDs. However, this means that the image being displayed impacts power consumption (e.g. a white image consumes more power than a black image as all the pixels are on). Prior OLED power management work has adapted the image being displayed through color inversion (white to black), changing the color scheme (to a darker color), or by using a gradient approach (darkening parts of the screen and showing only the required parts) [8]. These solutions work as studies $[2,3]$ have shown that it is possible to map one set of colors to another more power-efficient set without incurring significant end-user perceptual loss. Our approach builds on these techniques.

To do this, we use the concept of saliency. Saliency refers to where the user is looking at on the display. Explicitly, this is the part of the screen that commands the attention of the user. Saliency has been widely used in the field of image and video processing [6] where salient low level features such as color, orientation and intensity are used to discriminate the region-of-interest (ROI) from the rest of the visual content. Such approaches have also been used on mobile platforms [12], where it bridges the disconnect between the user's viewing attention with the salient image features to build a better Region-of-Interest (ROI) model that aids in the user viewing process. However, although such approaches are good in detecting ROIs in still images, it is computationally demanding and thus less useful for interactive systems such as games.

We use the approach proposed by El-Nasr et al. [4] that identifies the user's ROI heuristically instead of computationally identifying the precise ROI. This allows us to do the required ROI calculations fast enough to keep up with interactive games while still being able to successfully save overall power.

\section{DESIGN}

In this section, we list our design considerations. Our design focused on following requirements:

\section{Adaptive.}

The system needs to be adaptive to user interaction and adjust its power management accordingly. Prior work [7] has shown that users are usually unaware of both the power characteristics and the power saving settings off their smartphones. The system therefore must adapt and manage the power settings on behalf of the user.

\section{Exploit OLED characteristics.}

As mentioned earlier, OLED display power consumption depends greatly on the image being shown. To exploit this fact, we change the image/pixel properties of the screen (dimmer images consume less power).

\section{Usability and User Experience Consideration.}

The system should not affect the user's overall game experience - even when the power management is active. We tested this property with a user study (described in Section 6).

\subsection{Locus of Attention}

El-Nasr et al. [4], performed eye-tracking of users playing a FPS game (Halo II) and found that the main area of focus, within these games, was the center of the screen. Their study showed that the resolution of the main area of interest (the locus of attention) was approximately $300 \times 220$ pixels when the game resolution was $640 \times 480$ pixels. This locus was centered on the crosshair of the game located at the center of the screen (coordinate $\{320,240\}$ ).

For our study, we used a Nexus One phone that had an OLED display with a resolution of $800 \times 480$ pixels. We thus linearly scaled the results listed above and identified a locus of interested of around 300x180 pixels, centered at coordinate $\{400,240\}$ as shown in Figure 1.
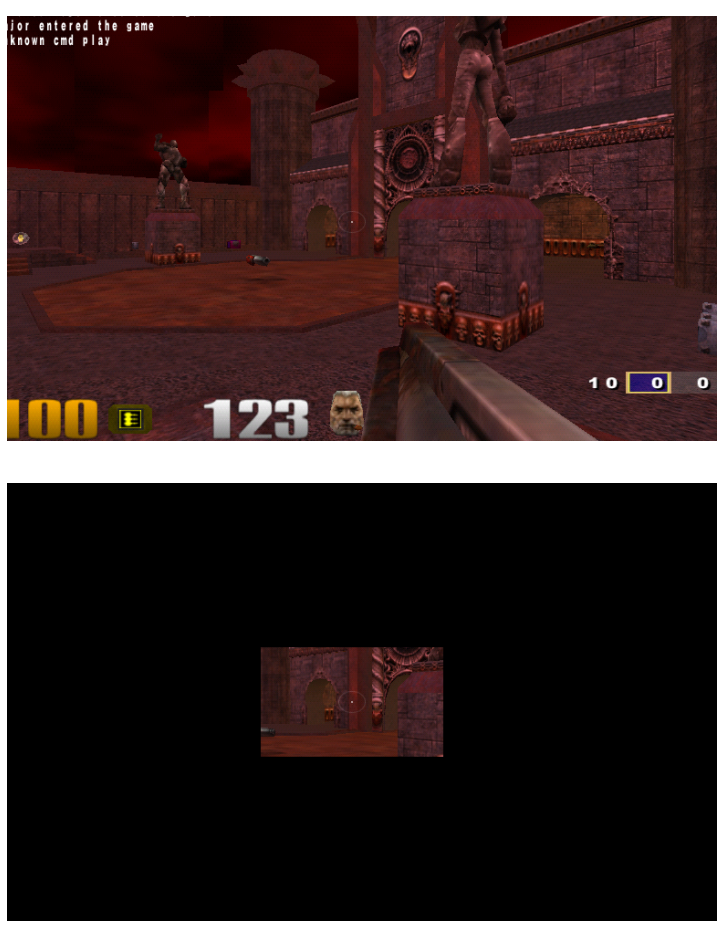

Figure 1: Locus of attention

The implication is that the remainder of the screen commands less interest to the user when playing the game. However, this does not imply that these non-locus areas are not useful during gameplay. Indeed, peripheral visual attention allows users to see the movement of artifacts as they come in and out of the locus of attention. Thus removing the non-locus areas completely, as shown in Figure 1, is not practical.

\subsection{Power Saving Technique}

To achieve a good balance between power saving and preserving the user's peripheral vision, we dim the areas outside the locus of attention - allowing us to achieve power savings. However, the challenge then becomes to affect this dimming 
without distracting the user (for example, naive dimming can can cause flickering as the user moves around).

To address this concern, we use a gradient dimming approach where areas close to the locus of attention are dimmed less than areas further away. We ensure that artifacts at the edge of the screen are still visible by ensuring that there is a minimum level of brightness that is applied to every pixel as shown in Figure 2. This minimum brightness level differs based on the OLED manufacturer and needs to be calibrated once for every new display (from a different manufacturer).
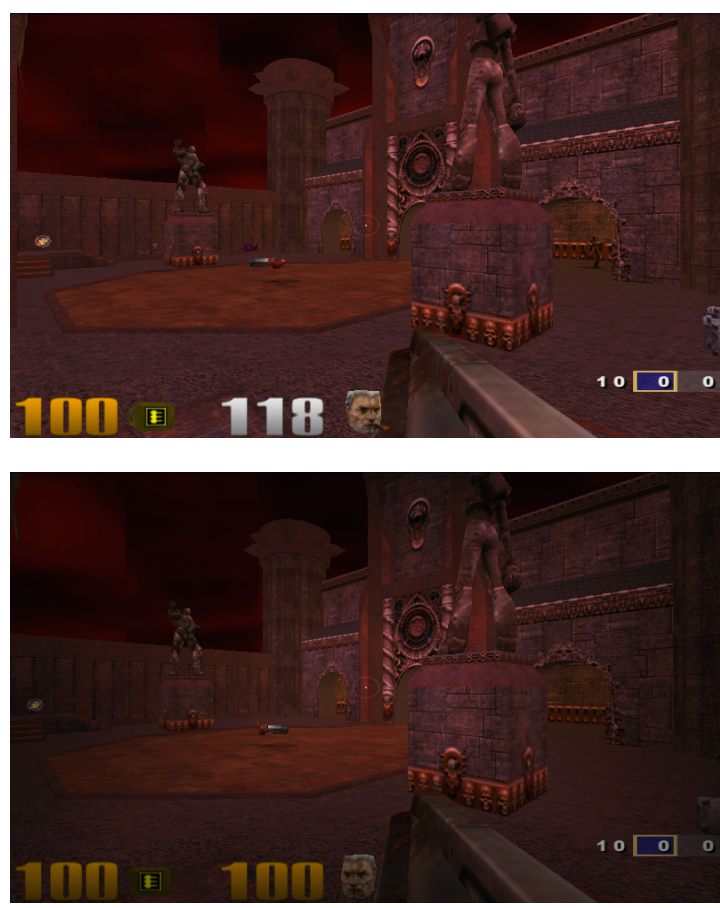

Figure 2: Gradual dimming from Locus of Attention to the edges

\subsection{Adapting to Game Play Situations}

The dimming mechanism can only be applied when the game is actually being played and even then, only in situations where the user's viewpoint is changing (either because the user is moving or looking around). When the user is stationary, we do not dim the display as prior work [4] has noted that the user's attention is spread across the entire screen when they are stationary.

\subsection{Adaptive Framework}

Our final implemented framework is shown in Figure 3 and implements the following: First, the system remains in normal power mode when the user is stationary within the game. Second, dimming is performed incrementally as the user initiate movements and vice versa (brightening the screen) as the user stops moving. This approach reduces user distraction as the user will not see the screen abruptly dim or brighten.

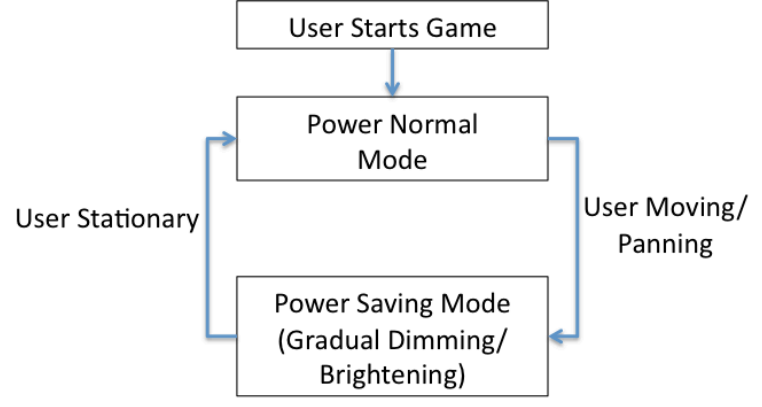

Figure 3: Adaptive Framework

\section{IMPLEMENTATION}

We tested our solution using Kwaak 3 [11], an Android version of the Quake 3 Arena [5] game. This game belongs to the First-Player Shooters (FPS) genre which demands both the most phone resources as well as the most user attention during gameplay. We believed these genre characteristics make Kwaak 3 an ideal test app and that any results achieved with Kwaak 3 can be generalized to other app categories.

\subsection{Kwaak 3 Overview}

Quake 3 Arena was developed by Id Software in 1999 and was a commercially successful FPS game. Id Software released the Quake 3 game engine under the GNU General Public License in 2005. It was then ported to Android and made publicly available by the Kwaak 3 [11] project. Kwaak 3 has two main game components: an app layer that sits on top of Android's Application Framework API and a native component developed using Android's Native Development Kit (NDK).

\subsection{Kwwak 3 Modification}

Our dimming technique utilizes the OpenGL API that is already present in Android's API stack. We achieve our dimming by performing alpha blending (to change the transparency of specific pixels) on the areas outside the locus of attention. To control the dimming, we added code to monitor the game's movement controls (the dimming is activated when the user moves and removed when the user stops). In particular, we monitored both the forward and backward controls mapped to the on-screen trackball as well as the pan controls that were mapped to screen gestures.

\subsection{Alpha Blending Implementation}

Alpha blending on the area outside the locus of attention is implemented as follows: First, we compute a series of dimming boxes from the locus of attention to the edge of the screen as shown in Figure 4. The maximum number of boxes is set to 60 . This number was derived experimentally so that each larger box still had the same aspect ratio as the screen (3:5). For the Nexus One (800x400 pixel display with a locus of attention of $300 \times 180$ pixels), this resulted in 60 boxes. In addition, we experimented with less than 60 boxes but found that the resulting images showed noticeable user artifacts (boundaries of the dimmed boxes becoming visible etc.).

Second, alpha blending is then applied to these boxed areas using the following heuristics. 1) The outermost box is 
set to the brightness level that is dim but still bright enough for the user to see. 2) The remaining 59 boxes (between the outermost box and the locus of attention) are then brightened linearly (with each box having a different brightness value) until the box next to the locus of attention is just a little dimmer than the locus. This heuristics ensures that we incrementally dim the screen from the locus of attention (brightest area) to the edge of the display (dimmest yet still visible area).

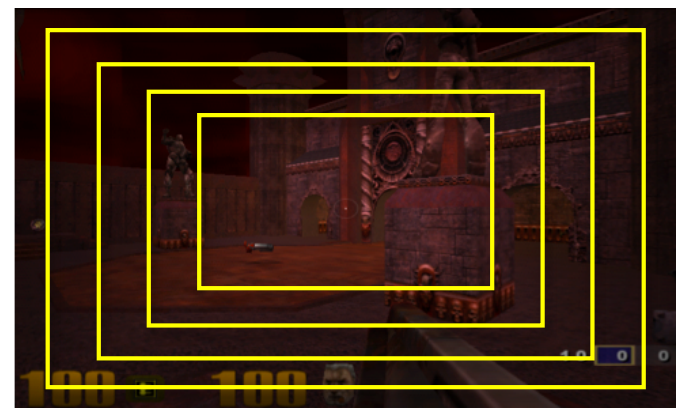

Figure 4: Dimming Boxes

\section{EVALUATION}

We focused on two evaluation goals: 1) To test the effectiveness of the power saving technique. In particular, how much power can we save, when playing a game, with our technique. 2) How playable is the game when our technique is active?. We tested both these goals via a user study involving 30 participants.

\subsection{Participant Selection and Devices}

The user study participants were elicited from the undergraduate and postgraduate population at Singapore Management University. The basic requirement for the user study was that each participant needed to be comfortable with smartphones. The evaluation was performed on a single Google Nexus One Android smartphone to ensure that the results were not skewed by inconsistent hardware or settings between phones (such as different OLED displays, OS version or settings).

In total, we obtained 30 participants for our user study (18 males, 12 females) who were within the age range of 16 to 27 . All our participants had prior experience with playing mobile games, with the majority preferring to play causal/puzzle mobile games. The majority indicated that they preferred to play mobile games during "quieter" moments when they were on public transport, queuing, or resting. The majority of the participants were also spent more than 15 minutes per day playing mobile games.

\subsection{Experiment Settings}

During the user study, each participant had to play two games of Kwaak 3, each lasting 15 minutes, that used the exact same map and configurations. One game used our technique and one did not. We used PowerTutor [13] to measure the component (display, CPU, network, etc.) power consumption of each game. The user was not told which version of the game they were trying and the order of the games (our version and normal) was counter-balanced between users to reduce bias.
To identify game playability issues, we tracked the number of kills (elimination of the enemy in the game) achieved by the user within the 15 minute playing window for both game versions. This provided an objective measure of whether players could achieved the same level of success with our version of the game (as compared to the default). We also surveyed each user to learn their qualitative opinion of the playability of each game version.

\subsection{User Study Procedure}

Each user study experiment was conducted in the following order. First, the participant was briefed on the project and motivation for this study. They then signed the consent form. Next, each participant was shown a demo of the unmodified Kwaak 3 game on the Google Nexus One smartphone. The participant was then allowed to try the game for a while to get used to the game mechanics, controls and map before the actual test. This allowed every participant to become somewhat familiar with the game even if they had no prior experience with it. After about 5 to 10 minutes of acclimatization, the actual test would begin.

After each game session, the user had to complete a questionnaire that asked them to rate the playability of that game. After completing both game sessions, the user had to fill up a final overall feedback form. Each user study took about one hour in total and each participant was paid USD $\$ 8$ for their participation. Finally, we did not influence the participants while they were playing the game - in particular, they were allowed to use whatever game play style (aggressive, cautious, camping, etc.) that they wished.

\section{RESULTS \& DISCUSSION}

In this section, we present and discuss the results from our user study.

\subsection{Power savings}

Figure 5 shows the power savings achievable by our method (The bars for "After" are with our technique turned on). We show both the total power savings as well as the savings for just the OLED power consumption. On average, across all participants, our methods managed to save about $11 \%$ of the OLED display power which resulted in a $4 \%$ total power savings.

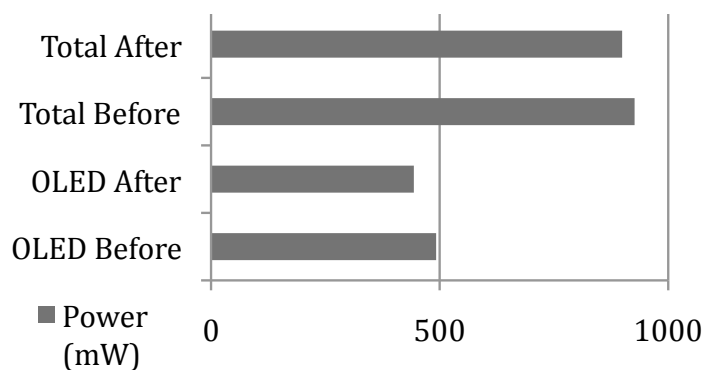

Figure 5: Power savings

\subsection{Playability}

To understand the playability impact of our solution, we reviewed the in-game performance (in terms of the number of "kills") of each player both with and without our system. 
The mean kill score across both both game variants, across all participants, was consistently low at 0.8 (original) and 1.2 (with our system) with a median of 0.5 and 1 respectively. This difference was statistically insignificant ( $\mathrm{p}$-value of 0.51 using a pairwise t-test). This indicated that our system had no impact on the player's kill totals. However, the low number of kills per player was a cause for concern as we expected many more kills to be obtained in a 15 minute game window.

To understand the reason for the low number of kills, we reviewed the survey feedback and noted that almost all the users had trouble with the controls for the game. In particular, most users ( $80 \%$ of the participant pool) found the game controls hard to master. They thus were unable to navigate or shoot effectively in the game and this contributed to their low kill scores. Hence, overall, we could not reach any definitive conclusion on the impact of our solution on the playability of the game.

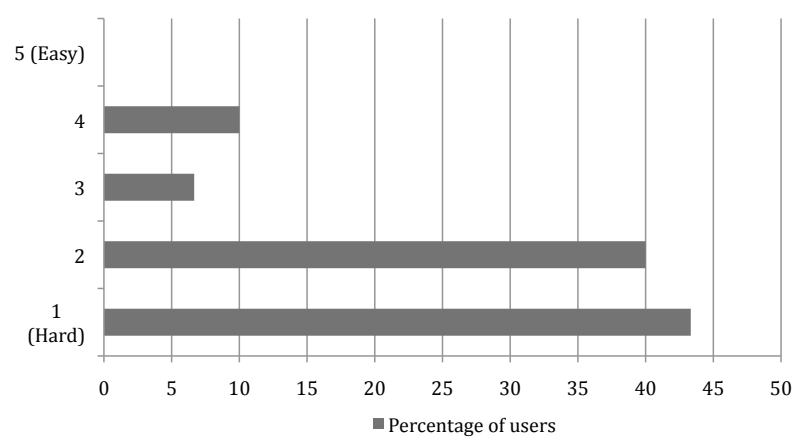

Figure 6: Ease of Controls

\subsection{Discussion}

In this section, we discuss the lessons learned during this study.

\subsubsection{The True Cost of Power Savings}

We could have significantly increased the OLED display power savings from $11 \%$ by dimming the rest of the screen extremely aggressively - albeit at the cost of playability. We believe that this tradeoff could be integrated within the game settings and provided as an option to the user - save minimal power with no playability impact or save more power with playability impact.

Our overall power savings is limited to $4 \%$ as we need additional CPU cycles to perform our alpha blending-based screen dimming. However, we believe that this CPU cost can be reduce by utilizing Android's native layer SDK (which provides power efficient image manipulation methods). Finally, another factor for our lower power savings was that quite a few participants (36\%) used a passive game play style where they did not move around much (possibly because they were not comfortable with the game controls). Our scheme would save more power for players who were constantly moving about the game world.

\subsubsection{Playability Has Many Aspects}

We found that our participant reported usability issues arose from Kwaak 3's controller implementation and not from our power dimming implementation. This was because
Quake 3 was never designed for mobile devices (that did not have a keyboard and mouse) and thus the movement controls had to be artificially mapped onto a few physical buttons, an on-screen joystick, and touch gestures.

As a result, our participants had major difficulties switching between the various control schemes when playing the game (moving around, shooting enemies, etc.). This resulted in the overall low number of kills per participants. Indeed, as noted above, $36 \%$ of the participants adopted a more passive game play style. We investigated the control issues in more detail and found that most participants found it much easier to track and engage enemies while they were stationary (Figure 7) compared to when they were moving (Figure 8). This was because the movement and tracking controls were laid out differently (movement used an on-screen joystick while tracking required screen swipes).

Overall, our experience is that there could be significant control issues inherent to playing complicated games on mobile devices. Hence, for our future tests, we will provide participants with external keyboards and mice to eliminate control issues.

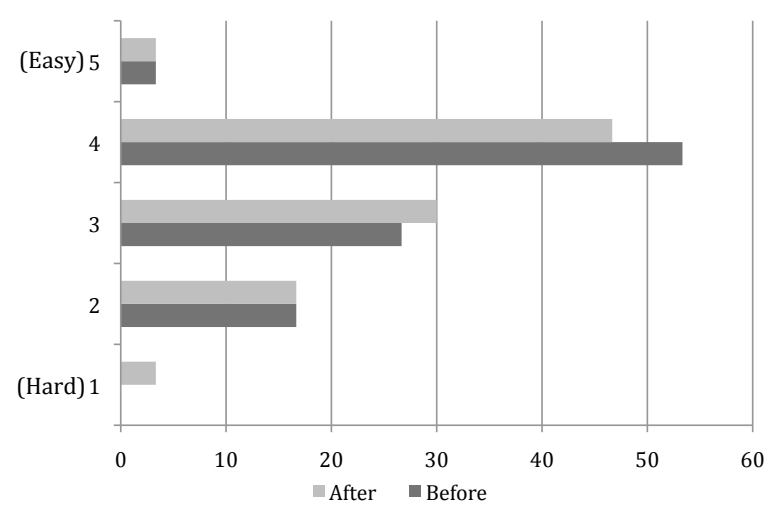

Figure 7: User ability to track when stationary

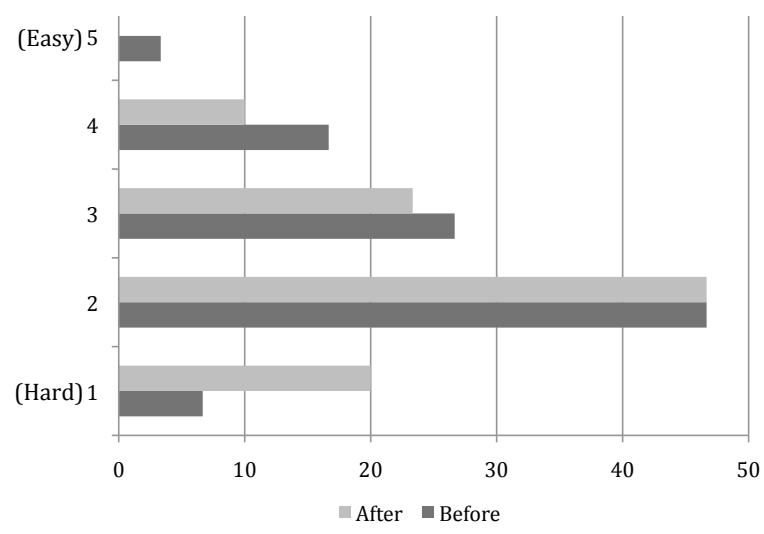

Figure 8: User ability to track when moving

\subsubsection{Generalizability of Technique}

The technique discussed so far was tested with one game using data from an eye tracking model developed for FPS games. In order to generalize it, we would need to first conduct additional eye tracking user studies to determine where 
mobile users look at when they are using applications from other genres on their mobile devices. We can then use this information to determine the appropriate locus of attention for other application genres. Next, we can make use of existing mobile OS features and notifications to improve our dimming mechanism. For example, we can perform gradual dimming when the user is scrolling (the faster the scroll, the dimmer it gets). We can also achieve very CPU-efficient dimming by implementing our mechanisms into the framebuffer pipeline of the mobile OS. By utilizing these behavioral and low-level OS features, we believe we can extend our dimming technique to many other applications. We plan to investigate these techniques in future work.

\section{CONCLUSION AND FUTURE WORK}

This work has demonstrated that we can save the power consumption of OLED displays, during game play, by gradually dimming the edges of screen when the user is moving. We implemented our solution on a Nexus One Android phone with the Kwaak 3 FPS game. We conducted a user study, involving 30 participants, to test our solution. The study showed that our method reduced the overall system power consumption by $4 \%$ without incurring and significant game playability impact.

In the future, we plan to address some of the issues and limitations found. This include resolving the controls issue by providing an external keyboard and mouse to participants or by using a game with a full touch interface (Kwaak 3 had a mix of touch gestures, on-screen joystick, and physical buttons). We also plan to move our processing framework into the Android native layer to reduce computational costs. Finally, moving forward, we envision that this work would become part of a larger bag-of-tricks approach where a number of techniques would come together to improve power savings on OLED-based mobile devices. For example, we could use touch occlusion (turning off off display areas under a finger that is touching the screen) to further improve the power savings. We can also use application context data, in this case, the game map and data about where "interesting" objects such as other players, doors, shops, etc. are located, to pro-actively dim the un-interesting areas (e.g. Increase the shadowing or dim the lights in those areas etc.). This will allow us to save additional power. We are currently investigating how to use this context-driven approach without impacting playability - smart players might realize that unusually dim areas are not "interesting".

\section{REFERENCES}

[1] B. Anand, K. Thirugnanam, J. Sebastian, P. G. Kannan, A. L. Ananda, M. C. Chan, and R. K. Balan. Adaptive display power management for mobile games. In Proceedings of the 9th international conference on Mobile systems, applications, and services, MobiSys '11, pages 57-70, New York, NY, USA, 2011. ACM.

[2] M. Dong, Y.-S. K. Choi, and L. Zhong. Power-saving color transformation of mobile graphical user interfaces on oled-based displays. In Proceedings of the 14th ACM/IEEE international symposium on Low power electronics and design, ISLPED '09, pages 339-342, New York, NY, USA, 2009. ACM.

[3] M. Dong and L. Zhong. Chameleon: a color-adaptive web browser for mobile oled displays. In Proceedings of the 9th international conference on Mobile systems, applications, and services, MobiSys '11, pages 85-98, New York, NY, USA, 2011. ACM.

[4] M. S. El-Nasr and S. Yan. Visual attention in 3d video games. In Proceedings of the 2006 ACM SIGCHI international conference on Advances in computer entertainment technology, ACE '06, New York, NY, USA, 2006. ACM.

[5] Id Software. Quake 3 Arena. http: //www.idsoftware.com/games/quake/quake3-arena/.

[6] L. Itti, C. Koch, and E. Niebur. A model of saliency-based visual attention for rapid scene analysis. Pattern Analysis and Machine Intelligence, IEEE Transactions on, 20(11):1254 -1259, nov 1998.

[7] A. Rahmati, A. Qian, and L. Zhong. Understanding human-battery interaction on mobile phones. In Proceedings of the 9th international conference on Human computer interaction with mobile devices and services, MobileHCI '07, pages 265-272, New York, NY, USA, 2007. ACM.

[8] P. Ranganathan, E. Geelhoed, M. Manahan, and K. Nicholas. Energy-aware user interfaces and energy-adaptive displays. Computer, 39(3):31 - 38, march 2006.

[9] The Nielsen Company. The State of Mobile Apps, Mar. 2011. http://blog.nielsen.com/nielsenwire/ online_mobile/the-state-of-mobile-apps/.

[10] The Nielsen Company. Who is Winning the U.S. Smartphone Battle?, Mar. 2011. http: //blog.nielsen.com/nielsenwire/online_mobile/ who-is-winning-the-u-s-smartphone-battle/?utm_ source $=$ feedburner\&utm_medium $=$ feed\&utm campaign=Feed:+NielsenWire+\%28Nielsen+Wire.

[11] thunderbird. Kwaak3. Android port of Quake 3 Arena. http://code.google.com/p/kwaak3/.

[12] X. Xie, H. Liu, S. Goumaz, and W.-Y. Ma. Learning user interest for image browsing on small-form-factor devices. In Proceedings of the SIGCHI conference on Human factors in computing systems, CHI '05, pages 671-680, New York, NY, USA, 2005. ACM.

[13] L. Zhang, B. Tiwana, Z. Qian, Z. Wang, R. P. Dick, Z. M. Mao, and L. Yang. Accurate online power estimation and automatic battery behavior based power model generation for smartphones. In Proceedings of the eighth IEEE/ACM/IFIP international conference on Hardware/software codesign and system synthesis, CODES/ISSS '10, pages 105-114, New York, NY, USA, 2010. ACM. 